\title{
BMJ Open Herbal medicine (Gan Mai Da Zao decoction) for depression: a systematic review protocol
}

\author{
Ji Hee Jun, ${ }^{1}$ Ju Ah Lee, ${ }^{1}$ Tae-Young Choi, ${ }^{1}$ Kyung-Jin Yun, ${ }^{1}$ Hyun-Ja Lim, ${ }^{2}$ \\ Myeong Soo Lee ${ }^{1}$
}

To cite: Jun JH, Lee JA, Choi T-Y, et al. Herbal medicine (Gan Mai Da Zao decoction) for depression: a systematic review protocol. BMJ Open 2014;4:e003690. doi:10.1136/bmjopen-2013003690

- Prepublication history for this paper is available online. To view these files please visit the journal online (http://dx.doi.org/10.1136/ bmjopen-2013-003690).

Received 30 July 2013 Accepted 20 November 2013

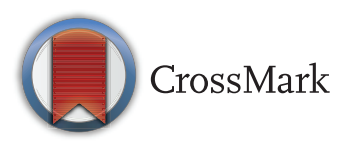

${ }^{1}$ Medical Research Division, Korea Institute of Oriental Medicine, Daejeon,

South Korea

${ }^{2}$ Department of Nursing, Chodang University, Muan, South Korea

Correspondence to Dr Myeong Soo Lee; drmslee@gmail.com

\section{ABSTRACT \\ Introduction: The aim of this systematic review is to analyse trial data on the effectiveness of a herbal medicine (Gan Mai Da Zao (GMDZ) decoction) in treating depression.}

Methods and analysis: 12 databases will be searched from their inception: PubMed, EMBASE, AMED, the Cochrane Library, five Korean medical databases (KoreaMed, DBpia, OASIS, the Research Information Service System (RISS) and the Korean Studies Information Service System (KISS)) and three Chinese medical databases (China National Knowledge Infrastructure (CNKI), the Wanfang Database and the Chinese Scientific Journals Database (VIP)).

Randomised clinical trials (RCTs) or quasi-RCTs using a GMDZ decoction for any type of depression will be considered. The selection of the studies, data abstraction and validations will be performed independently by two researchers.

Dissemination: The findings will be disseminated to appropriate audiences via peer-reviewed publication and conference presentations.

Trial registration number: PROSPERO 2013: CRD42013005100.

\section{INTRODUCTION}

\section{Description of the condition}

The prevalence of depression is as high as $10 \%$ in the population, with half of the affected people having recurrent symptoms. ${ }^{1}$ Depression is the most common clinical mental disorder and is characterised by emotional depression, a loss of interest or pleasure, insomnia, increased dreaming, a loss of appetite, feelings of worthlessness or excessive guilt, and recurrent thoughts of death or suicide. $^{2}{ }^{3}$ This disorder is a common and debilitating condition with a pervasive impact on the quality of life of both patients and their families. ${ }^{4}$ Additionally, depression is a serious mental illness, with a high morbidity rate, a high relapse rate, a high disability rate, a high suicide rate and strong negative effects on people's health. According to the WHO,

\section{Strengths and limitations of this study}

- The strength of this systematic review is its extensive, unbiased search of various databases without language restrictions.

- Our review will give readers the opportunity to access studies originally published in East Asian languages that they would otherwise be unable to read.

- Our review will be useful to practitioners and patients regarding the use of GMDZ decoction in depression treatment.

depression is the leading cause of disability for all ages and both sexes worldwide, with an estimated 350 million people affected, ${ }^{5}$ and in 2020, depression will become the second largest burden of disease after heart attack. ${ }^{3}$ Therefore, we must find effective treatment methods for patients and the society.

\section{Description of the intervention}

The Gan Mai Da Zao (GMDZ) decoction is one of the most well-known herbal prescriptions for depression, and its use was first documented in the Jin Gui Yao Lue (Synopsis of Prescriptions of the Golden Chamber), a classical Chinese medical book by $\mathrm{Dr}$ Zongjing Zhang (AD 152-219). ${ }^{6}$ The composition of GMDZ decoction includes the three herbs Glycyrrhiza, Triticum and Zizyphi Fructus. Many pharmaceutical companies around the world manufacture GMDZ to manage depression and emotional disturbances. They are regulated as pharmaceutical drugs in China, Japan, Korea and Taiwan and as dietary supplements in North America. Several forms of GMDZ are available, such as extracts, tablets, capsules, pills, powders or injections. The composition of commercial GMDZ/100 g is generally $16.1 \mathrm{~g}$ Glycyrrhiza, $64.5 \mathrm{~g}$ Triticum and $19.4 \mathrm{~g}$ Zizyphi Fructus and the dosage is $2.00-3.25 \mathrm{~g} /$ day for adults. Physicians have used this formula 
for depression and for emotional problems. Several studies have demonstrated the antidepressant effects of the GMDZ decoction. ${ }^{78}$

\section{How the intervention might work}

GMDZ may improve depression symptoms by reducing glutamate levels and by increasing the $N$-methyl-Daspartate receptor (NR) subunits NR2A and NR2B in the frontal cortex and hippocampus. ${ }^{9}$ The GMDZ decoction had beneficial effects on behaviour and brain monoamine neurotransmitter levels (NE and 5-HT) in a depression model. ${ }^{10}{ }^{11}$ However, further studies are required to identify the exact mechanisms of action.

\section{Why it is important to this review}

Currently, the GMDZ decoction alone or combined with antidepressant drugs is widely used as an effective alternative method for depression treatment in China. However, there is no critically appraised evidence, such as a systematic review or meta-analyses, of the potential benefits and harm of the GMDZ decoction in depression. Therefore, this systematic review aims to summarise and critically evaluate the evidence from clinical trials that have tested the effectiveness of the GMDZ decoction as a treatment for depression, either alone or in combination with other antidepressant drugs, compared with antidepressant drugs alone.

\section{METHODS}

Criteria for considering studies for this review

Types of studies

All prospective randomised clinical trials (RCTs) and quasi-RCTs (RCTs in which the allocation to treatment was obtained by alternation, the use of alternate medical records, date of birth or other predictable methods) will be included in this systematic review. All evaluations of the use of the GMDZ decoction for the treatment of depression will be included in this review. Case studies, case series, qualitative studies and uncontrolled trials will be excluded. Trials that failed to provide detailed results will also be excluded. Dissertations and abstracts will be included if these documents contain sufficient detail for critical evaluation. No language restrictions will be imposed. If we encounter languages other than English, Korean and Chinese, we will either contact the original authors or translate the manuscript using a professional service.

\section{Types of participants}

Patients of any age and sex with depression (comorbidity), diagnosed based on the Diagnostic and Statistical Manual (DSM-IV) ${ }^{12}$ the International Classification of Mental and Behavioural Disorders (ICD-10) ${ }^{13}$ or the Criteria for Classification and Diagnosis of Mental Diseases (CCMD-3-R $)^{14}$ will be included.
Types of interventions

Studies that used a GMDZ decoction or a modified GMDZ decoction will be included. A GMDZ decoction includes the following three herbs: Glycyrrhiza, Triticum and Zizyphi Fructus. We will include all forms of this medication, such as extracts, tablets, capsules, pills, powders or injections. The modified GMDZ decoction formula is defined by practitioners as a decoction with no more than one herb of the three original herbs, but which has almost the same actions as the original GMDZ decoction formula. Trials of combined interventions with other decoction treatments will be excluded. Trials in which the GMDZ decoction was used as an adjunct to conventional treatment (treatments that are widely accepted and used by the mainstream medical community, including antidepressants), usual care (which can include routine care received by patients for prevention or treatment of disease) or standard care (the ordinary level of skill and care that any healthcare practitioner would be expected to observe in caring for patients) will also be included if the control group received the same concomitant treatments as the GMDZ decoction group. The control groups will include antidepressant drug use, placebo use, no treatment and usual care or standard care.

\section{Types of outcome measures}

For inclusion, data on at least one primary outcome need to be reported.

\section{Primary outcomes}

1. Treatment efficacy: the number of patients whose depression symptoms improved

2. Severity of depression (self-reported scale) with the Beck Depression Inventory ${ }^{15}$ and the Hamilton Rating Scale for Depression ${ }^{16}$

\section{Secondary outcomes}

1. Quality of life

2. Adverse events

\section{Search methods for the identification of studies}

Electronic searches

The following databases will be searched from their inception: PubMed, EMBASE, AMED, the Cochrane Library, five Korean medical databases (KoreaMed, DBpia, OASIS, the Research Information Service System (RISS) and the Korean Studies Information Service System (KISS)) and three Chinese medical databases (China National Knowledge Infrastructure (CNKI), the Wanfang Database and the Chinese Scientific Journals Database (VIP)).

\section{Other sources}

Studies will also be obtained from the following sources:

- The reference lists of all relevant articles;

- Hand searching of department files;

- Unpublished conference proceedings relevant to depression will be reviewed, if available. 


\section{Search strategy}

The search will be conducted in Korean, Chinese and English using the following terms: (depression OR Melancholia OR major depression OR poststroke depression OR postpartum depression OR involutional depression OR climacteric depression OR senile depression) AND (gan mai da zao OR gan mai da zao tang OR ganmai dazao OR ganmai dazao tang OR gan mai da zao* OR modified gan mai da zao OR modified ganmai dazao OR kambakutaisoto OR kam baku tai soto). These strategies will be modified for use with other databases.

\section{Data collection and analysis}

Selection of studies

All of the titles and abstracts of studies retrieved with the electronic searches will be reviewed by three authors (JHJ, JAL and T-YC), who will select relevant articles by title and abstract. Hard copies of each publication will be reviewed by three independent authors to determine their inclusion based on the inclusion criteria (JHJ, JAL and T-YC). Any disagreements will be resolved through discussion by three authors (JHJ, T-YC and K-JY) and an arbiter (MSL). We will contact the authors of the included studies for clarification if necessary. The details of the study selection are shown in a PRISMA flow chart (figure 1).
Data extraction and management

Two authors (JHJ and T-YC) will extract data from the selected reports or studies and independently complete a data collection form. We will use GRADEpro software in the Cochrane Systematic Reviews to create a Summary of Findings table. In addition, information such as the participants, interventions, outcomes and results will be obtained from each report. Any disagreement between the two authors will be resolved by discussion. Another author (MSL) will act as an arbiter for unresolved disagreements.

\section{Assessment of risk of bias in included studies}

According to the guidelines of the Cochrane Handbook of Systematic Reviews of Interventions, the risk of bias will be assessed to evaluate the methodological quality of the included studies. ${ }^{17}$ The following domains will be evaluated for methodological quality: sequence generation, allocation concealment, blinding of participants and outcome assessors, incomplete outcome data and selective outcome reporting. The evaluated domains will be judged as 'Low', 'High' or 'Uncertain' according to the criteria of the Cochrane guidelines.

\section{Measures of treatment effect}

Dichotomous data will be presented as a risk ratio (RR) with $95 \%$ confidence intervals (CIs). For continuous
Figure 1 Flow diagram of the trial selection process. NRS, non-randomised studies.
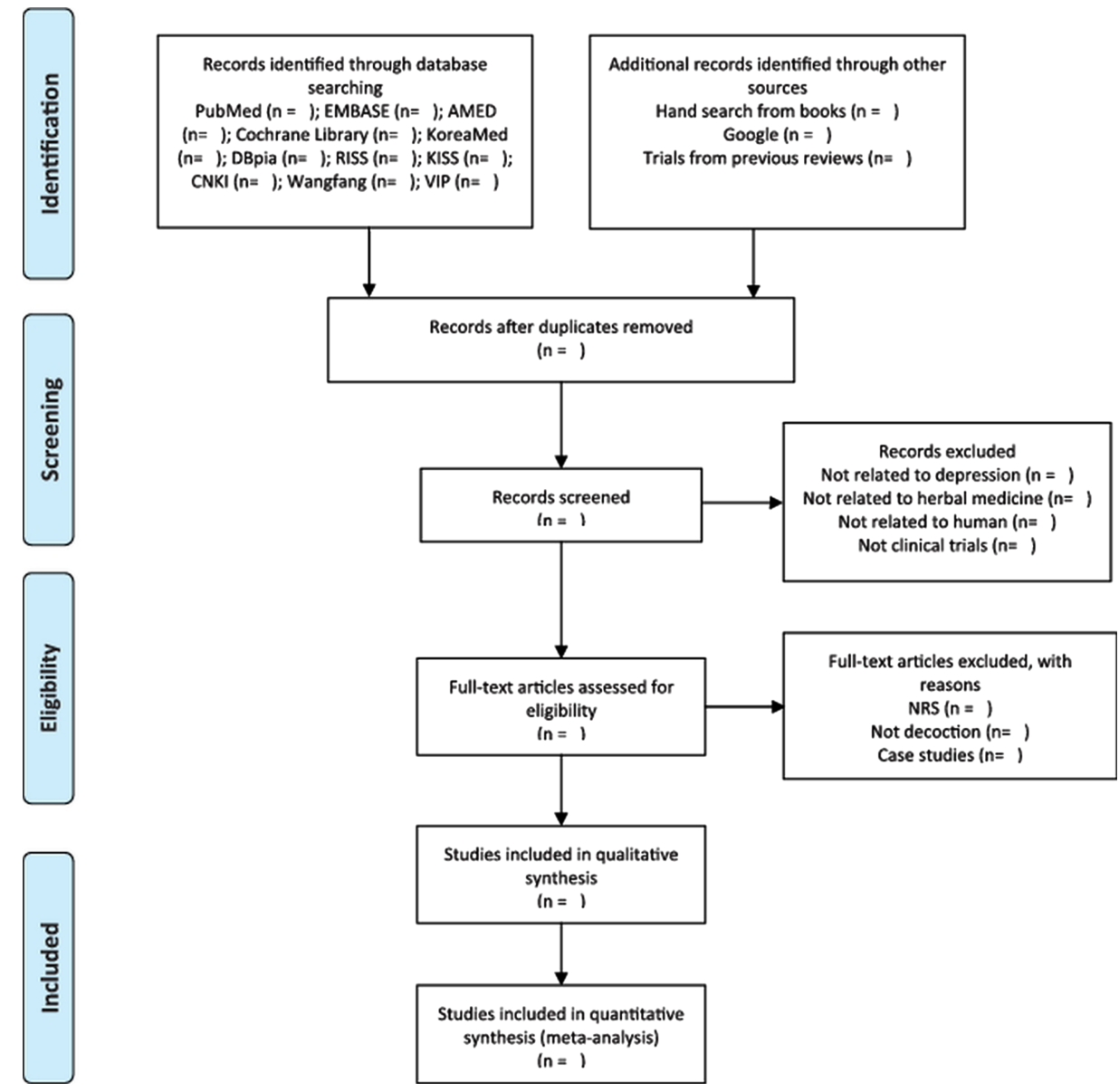
data, the mean difference (MD) will be used with $95 \%$ CIs. In cases of outcome variables with different scales, the standard MD will be used instead of the weighted MD.

\section{Unit of analysis issues}

The meta-analysis will include data from parallel-group design studies. In the case of cross-over trials, we will use the first phase of the data. If there are multiple timepoint observations, the data will be analysed as either short-term (within 4 weeks) or long-term (over 4 weeks) follow-up.

\section{Dealing with missing data}

If missing data are detected, we will request any missing or incomplete information from the original study investigators.

\section{Assessment of heterogeneity}

A fixed-effects and a random-effects models will be simultaneously used for the meta-analysis. Heterogeneity will be tested with the Higgins $\mathrm{I}^{2}$ test. We will calculate the $\mathrm{I}^{2}$ statistic, which will provide a measure of the inconsistencies among the included studies. We will use a $50 \%$ cut-off point for meaningful heterogeneity among the included studies. If heterogeneity is observed, subgroup analysis will be conducted. ${ }^{18}$

\section{Assessment of reporting biases}

Funnel plots will be used to detect reporting biases and small-study effects. If more than 10 studies are included in the meta-analysis, the test for funnel plot asymmetry will be conducted using Egger's method. ${ }^{19} 20$

\section{Data synthesis}

If a significant number of studies are identified, a meta-analysis will be conducted with simultaneous use of fixed-effect and random-effect models. All statistical analyses will be performed using RevMan V.5.1 (the Cochrane Collaboration) software. To summarise the effects of a GMDZ decoction on outcomes (response rate), we will abstract the risk estimates (RR), and 95\% CIs using Review Manager V.5.0 for Windows (Cochrane Collaboration's software). For studies with insufficient information, we will contact the primary authors to acquire and verify the data where possible. If appropriate, we will then pool the data across studies using random effect models.

\section{Subgroup analysis and investigation of heterogeneity}

To explore differences in effect sizes, subgroup analyses will be conducted on the following topics: the severity of depression, sex, the type of GMDZ decoction, the design of the trial (eg, cross-over group or parallel group), the dose of GMDZ decoction and treatment frequency. We will also summarise the standardisation and characteristics of GMDZ decoctions from all included studies.
Sensitivity analysis

Sensitivity analysis will be conducted according to the following criteria:

1. Methodological qualities (sequence generation, allocation concealment or blinding);

2. Sample size (small sample studies, eg, less than 40 participants in each group or large sample studies, eg, more than 40 participants in each group).

\section{DISCUSSION}

This systematic review will provide a detailed summary of the current state of evidence on the effectiveness of the herbal medicine GMDZ decoction in treating the symptoms of patients with depression. The review will benefit patients and practitioners in the field of traditional and complementary medicine.

Contributors The protocol was drafted by JHJ, JAL, T-YC, K-JY, H-JL and MSL. The search strategy was developed and will be run by JHJ and JAL. Copies of studies will be obtained by JHJ, JAL and T-YC. Selection of the studies to include will be carried out by JHJ, JAL and T-YC. MSL will act as an arbiter in the study selection stage. Extraction of data from studies will be conducted by JHJ, JAL and T-YC. Entering data into RevMan will be conducted by JAL and K-JY. Carrying out the analysis will be performed by $\mathrm{JHJ}, \mathrm{JAL}$ and MSL. Interpretation of the analysis will be carried out by $\mathrm{JHJ}$, T-YC, K-JY and MSL. The final review will be drafted by JHJ, JAL, T-YC, K-JY, $\mathrm{H}-\mathrm{JL}$ and MSL. The review will be updated by JHJ and MSL.

Funding None.

Competing interests The authors were supported by Korea Institute of Oriental Medicine (K13400, K13130, K13281).

Provenance and peer review Not commissioned; externally peer reviewed.

Open Access This is an Open Access article distributed in accordance with the Creative Commons Attribution Non Commercial (CC BY-NC 3.0) license, which permits others to distribute, remix, adapt, build upon this work noncommercially, and license their derivative works on different terms, provided the original work is properly cited and the use is non-commercial. See: http:// creativecommons.org/licenses/by-nc/3.0/

\section{REFERENCES}

1. Cipriani A, Barbui C, Butler R, et al. Depression in adults: drug and physical treatments. Clin Evid 2011;2011:1003.

2. World Health Organization. Depression: definition. http://www.euro. who.int/en/what-we-do/health-topics/noncommunicable-diseases/ sections/news/2012/10/depression-in-europe/depression-definition. (accessed 20 Nov 2013).

3. World Health Organization. Mental health: depression in Europe. http://www.euro.who.int/en/what-we-do/health-topics/ noncommunicable-diseases/mental-health/news/news/2012/10/ depression-in-europe (accessed 20 Nov 2013).

4. Wray NR, Pergadia ML, Blackwood DH, et al. Genome-wide association study of major depressive disorder: new results, meta-analysis, and lessons learned. Mol Psychiat 2012;17:36-48.

5. World Health Organization. Depression. http://www.who.int/topics/ depression/en/ (accessed 20 Nov 2013).

6. Ruan J. Chinese-english synopsis of prescription of the Golden Chamber. Shanghai, China: Shanghai Science and Technology Publishing Company, 2003.

7. Wu J. Antidepressant effect of gan-mai-da-zao tang on depression compared with fluoxetine. J Chin Physician 2002;30:18-19.

8. Yang FE, Qiao QF, Gong YP. Antidepressant effect of gan-mai-da-zao tang on 30 women with postpartum depression. Shanxi J TCM 2009;30:851-52.

9. Lou JS, Li CY, Yang XC, et al. Protective effect of gan mai da zao decoction in unpredictable chronic mild stress-induced behavioral and biochemical alterations. Pharm Biol 2010;48:1328-36. 
10. Zhang Q, Yang J, Liu YX, et al. Influence on neurotransmitter in brain of depressed model rat treated by Bai-di-gan-zao decoction. $J$ Chengdu Univ TCM 2006;29:21-3.

11. Zhang $\mathrm{XL}$, Dai WW, Jin GQ, et al. Changes of gene expression profile of receptors \& ion channels in hippocampus of rat model with depression and adjusting effects of supplemented ganmai dazao decoction. Pharmacol Clin Chin Mater Med 2011;27:6-10.

12. American Psychiatric Association. Diagnostic and statistical manual of mental disorders. 4th edn. Washington, DC: American Psychiatric Association, 1994.

13. World Health Organisation. The ICD-10 Classification o mental and behavioural disorders. Diagnostic criteria for research. Geneva: World Health Organisation, 1993.

14. Chinese Psychiatric Society. The Chinese classification of mental disorders [CCMD-3]. Shandong, China: Shandong Publishing House of Science and Technology, 2001.

15. Beck AT, Ward $\mathrm{CH}$, Mendelson $\mathrm{M}$, et al. An inventory for measuring depression. Arch Gen Psychiatry 1961;4:561-71.
16. Hamilton M. A rating scale for depression. J Neurol Neurosurg Psychiatry 1960;23:56-62.

17. Higgins JPT, Altman DG, Sterne JAC. Chapter 8: assessing risk of bias in included studies. In: Higgins JPT, Green S, eds. Cochrane handbook for systematic reviews of interventions version 5.1.0 (updated March 2011). The Cochrane Collaboration. http://www. cochrane-handbook.org, 2011.

18. Deeks JJ, Higgins JPT, Altman DG. Chapter 9: analysing data and undertaking meta-analyses. In: Higgins JPT, Green S, eds. Cochrane handbook for systematic reviews of interventions version 5.1.0 (updated March 2011). The Cochrane Collaboration. http://www.cochrane-handbook.org, 2011.

19. Egger M, Davey Smith G, Schneider M, et al. Bias in meta-analysis detected by a simple, graphical test. BMJ 1997;315:629-34.

20. Sterne JAC, Egger M, Moher D. Chapter 10: addressing reporting biases. In: Higgins JPT, Green S. eds. Cochrane handbook for systematic reviews of interventions version 5.1.0 (updated March 2011). The Cochrane Collaboration, 2011. http://www. cochrane-handbook.org 